\title{
Translocating lipopolysaccharide correlates with the severity of enterovirus A71-induced HFMD by promoting pro-inflammation and viral IRES activity
}

\section{Yuya Wang}

Chongqing Medical University

\section{Xiaoling Xue}

Chongqing Medical University

\section{Kena Dan}

Chongqing Medical University

\section{Xiongbo Yang}

Chongqing University

\section{Qingqing Yang}

Changzhi Medical College Affiliated Heping Hospital

\section{Xujiao Feng}

Changzhi Medical College Affiliated Heping Hospital

Jing Yang

Chongqing University

\section{Bangtao Chen ( $\square$ medisci@163.com )}

Chongqing University https://orcid.org/0000-0001-6540-8726

\section{Research}

Keywords: Hand, foot, and mouth disease, Enterovirus A71, 2A protease (2A1'), Lipopolysaccharide, Inflammation, internal ribosomal entry site (IRES)

Posted Date: August 17th, 2021

DOl: https://doi.org/10.21203/rs.3.rs-806112/v1

License: (c) (1) This work is licensed under a Creative Commons Attribution 4.0 International License. Read Full License

Version of Record: A version of this preprint was published at Gut Pathogens on November 22nd, 2021. See the published version at https://doi.org/10.1186/s13099-021-00465-x. 
Title: Translocating lipopolysaccharide correlates with the severity of enterovirus

3 A71-induced HFMD by promoting pro-inflammation and viral IRES activity

Authors: Yuya Wang ${ }^{1}$, Xiaoling Xue ${ }^{2}, K$ Kena Dan $^{3}$, Xiongbo Yang ${ }^{4}$, Xujiao Feng ${ }^{5}$,

1 Department of Obstetrics and Gynecology, the Third Affiliated Hospital of

Chongqing Medical University, Chongqing, 401120, China

2 Department of Hematology, the Third Affiliated Hospital of Chongqing Medical

University, Chongqing, 401120, China

3 Department of Dermatology, the Third Affiliated Hospital of Chongqing Medical

University, Chongqing, 401120, China

Chongqing, 404100, China

5 Department of Infectious diseases, Heping Hospital Affiliated to Changzhi Medical

College, 046000, China

*Correspondence: feijing_2002@163.com; medisci@163.com

Running Title: LPS Aggravates EV-A71-induced HFMD

Key words: Hand, foot, and mouth disease; Enterovirus A71; 2 A protease $\left(2 \mathrm{~A}^{\mathrm{pro}}\right)$; 
Background: The increase of inflammation-inducing enterobacteria was recently observed in severe hand, foot, and mouth disease (HFMD) caused by Enterovirus A71 (EV-A71). This study aimed to verify the occurrence of bacterial translocation (BT) and further explore the contributory role of BT to severity of EV-A71-mediated HFMD cases.

Methods: Serum specimens from 65 mild and 65 severe EV-A71-associated HFMD cases and 65 healthy children were collected. EV-A71 VP1 in serum, inflammatory mediators including C-reactive protein, IL-1 $\beta$, IL-6, interferon- $\gamma$ and tumor necrosis factor- $\alpha$, BT related biomarkers including Claudin-3, intestinal fatty acid binding protein, lipopolysaccharide (LPS), soluble CD14(sCD14) and endotoxin core antibody were measured by ELISA. Bacterial DNA (BactDNA) fragments were quantified by quantified PCR (qPCR). Rhabdomyosarcoma (RD) cells, infected with LPS-pre-incubated EV-A71 or transfected with plasmid containing viral $2 \mathrm{~A}^{\text {pro }}$ or mRNA containing viral internal ribosomal entry site (IRES), were post-treated with or without LPS in vitro. EV-A71 RNA and viral or cellular proteins were determined by qPCR and western blot, respectively.

Results: Compared to mild HFMD patients, remarkably higher inflammatory mediators as well as BT-related biomarkers except BactDNA were observed in severe HFMD cases (all $P<0.05$ ). In severe HFMD group, circulating concentrations of LPS and sCD14 showed statistical correlations with inflammation indices (all $P<0.05$ ), serum levels of EV-A71 VP1 were found to be positively correlated with serum LPS 
60

61 63 65

62 64 66

$(r=0.341, P=0.005)$ and serum $\mathrm{SCD} 14(r=0.458, P<0.001)$. In vitro, $\mathrm{EV}-\mathrm{A} 71$

attachment and internalization were only slightly promoted by LPS pre-incubation;

however, EV-A71 proliferation and viral $2 \mathrm{~A}^{\text {pro }}$-mediated IRES activity were significantly accelerated by LPS post-treatment.

Conclusions: Our results collectively indicate that gut-derived translocating LPS

contributes to the severity of EV-A71-induced HFMD by driving inflammatory response and viral proliferation via viral $2 \mathrm{~A}^{\mathrm{pro}}$-mediated IRES.

3

54

55

5

(1)

58

9

60

\section{1}




\section{Background}

Enterovirus A71 (EV-A71) is well known to be the major etiological culprit causing hand, foot, and mouth disease (HFMD) in children aged five and below. EV-A71-associated HFMD generally presents as a self-limiting illness. However, some patients may rapidly develop neurological complications and cardiopulmonary disorders that occasionally even cause death. In 1969, Schmidt et al isolated the first strain of EV-A71 from the stool samples of children with disease of the central nervous system in California, USA [1]. Since then, several outbreaks of EV-A71 infection have been reported across the Asia-Pacific region. In China, it caused the death of 479 children during 2008-2009 and more than 1 million cases per year have been monitored since 2008 [2, 3]. Note worthily, three inactivated monovalent EV-A71 vaccines were licensed in China in 2016; however, the vaccines are only available in the private market in China and the vaccines' effectiveness against severe HFMD remains yet unknown [4]. And to date, few established antiviral therapies are available for severe EV-A71 infection. Collectively, EV-A71-associated HFMD (especially the severe conditions) still pose a growing global public health and economic concern in affected areas.

EV-A71 is a non-enveloped, positive-sense, single-stranded RNA virus that belongs to genus Enterovirus in the family Picornaviridae. Structurally, the icosahedral virus particle harbors a RNA genome of approximately $7.4 \mathrm{~kb}$ in size with two open reading frames, which is flanked by a highly structured 5'-untranslated region (5'UTR) and a 3'UTR with a poly (A) tail [5]. EV-A71 5'UTR contains a type I 
internal ribosomal entry site (IRES) mediating initiation of viral proteins translation.

By IRES-driven translation, four structural viral proteins (VP1-VP4) and seven non-structural viral proteins $(2 \mathrm{~A}-2 \mathrm{C}$ and $3 \mathrm{~A}-3 \mathrm{D})$ are finally synthesized with the cis-cleavage actions of viral proteases $\left(2 \mathrm{~A}^{\text {pro }}\right.$ and $\left.3 \mathrm{C}^{\mathrm{pro}}\right)[6]$.

For the survival of EV-A71 in host, viral proteases are the most important promoters for evading host's antiviral innate immunity by hijacking host cell cap-dependent translation via hydrolysis of eukaryotic initiation factor 4GI (eIF4GI) and other cellular proteins[7]. Although the exact pathogenesis of severe HFMD caused by EV-A71 has not been fully elucidated, increasing evidence have shown that

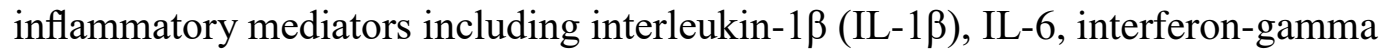
(IFN- $\gamma$ ), C-reactive protein (CRP), tumor necrosis factor- $\alpha$ (TNF- $\alpha$ ), etc. contribute to the development and severity of EV-A71-associated HFMD in Children [8, 9]. The potential mechanism of systemic inflammation activation accompanied by EV-A71 infection has not yet been determined; a recent study preliminarily demonstrated that the up-regulation of inflammation-inducing enterobacteria may be the prevailing cause for severity of HFMD [10].

Enteric dysbacteriosis along with increased intestinal mucosal permeability will result in a higher translocation rate of microbial immunogenic components from the gut into the circulatory system, which is the so-called bacterial translocation (BT). Serological indicators of leaky gut including Claudin-3 and intestinal fatty acid binding protein (I-FABP), bacterial components including lipopolysaccharide (LPS) and bacterial DNA (BactDNA), products to LPS challenge including soluble CD14 
111

112

(sCD14) and endotoxin core antibody (EndoCAb) are usually applied to evaluate BT [11-17]. Clinically, BT was proved to be associated with systemic inflammation in patients with cirrhosis [13], psoriasis [14], inflammatory bowel disease [15], hepatitis virus and human immunodeficiency virus infection $[16,17]$. However, occurrence of leaky gut-related BT and its association with exacerbation of inflammatory response in HFMD children are poorly investigated.

Taken together, we hypothesize that pro-inflammation cytokines characterize the severity of HFMD and increased intestinal permeability-caused BT is one of main culprits for tuning process of inflammation. With regard to this, in present study, we focused on the correlations between leaky gut-related BT and inflammation-driven severity of HFMD, and further assessed the possible mechanism of BT in EV-A71 infection, in the hope of providing more convincing evidence for BT-derived inflammatory pathogenesis of HFMD deterioration.

\section{Materials and methods}

\section{Subjects}

This study was approved by and carried out under the guidelines of the Ethics Committee of Heping Hospital affiliated to Changzhi Medical College. Before enrollment, informed consent was obtained from the parents/guardians of all the recruited children. Total 130 EV-A71-induced HFMD patients (65 mild cases and 65 severe cases) and 65 age- and gender-matched healthy children were collected during 2015 to 2018 in Heping Hospital. All the patients were etiologically confirmed by 
EV-A71 RNA detection in stool or throat swabs. According to the Chinese guidelines for the diagnosis and treatment of HFMD (2018 edition) [18], severe HFMD cases were clinically diagnosed if they experienced any neurological complications and/or cardiopulmonary complications. Children with other comorbidities such as juvenile idiopathic arthritis etc. or medications such as systemic anti-inflammation are excluded.

\section{Laboratory examination}

Peripheral blood samples were collected from all the subjects. Blood cell count and liver function were routinely examined. The protein levels of indicators assessed by enzyme-linked immunosorbent assay (ELISA) in present study involved CRP (\#E007462, 3ABio), IL-1 $\beta$ (\#E001772, 3ABio), IL-6 (\#E000482, 3ABio), IFN- $\gamma$ (\#C608-01,GenStar), TNF- $\alpha$ (\#489204, Cayman), LPS (\#DG11072H, Dogesce), I-FABP (\#DFBP20, R\&D Systems), Claudin-3 (\#abx250611, Abbexa), sCD14 (\#DC140, R\&D Systems) and EndoCAb (\#E013362, 3ABio). Human EV-A71 VP1 protein ELISA kit (\#MM-13481H2, MeiMian) was applied to detect the protein level of EV-A71 VP1 from blood serum. Assays were performed according to the manufacturer's specifications and the detection limits were in line with the manufacturer's instructions. All the plates were read by the I Mark ${ }^{\mathrm{TM}}$ Micro plate Reader (BIO-RAD).

\section{Cell culture, virus infection, transfection, stimulation and luciferase assay}

Human rhabdomyosarcoma (RD) cells (ATCC® CCL-136) were maintained in Dulbecco's modified Eagle's medium (DMEM, Gibco) containing 10\% fetal bovine 
155

156

157

serum (FBS, Hyclone) with $100 \mathrm{U} / \mathrm{mL}$ penicillin and $100 \mu \mathrm{g} / \mathrm{mL}$ streptomycin. The cells were infected with EV-A71 (ATCC® VR-1432TM, BrCr strain) at the multiplicity of infection (MOI) of 2 or transfected with N-terminal GFP-tagged EV-A71 2A expression plasmid and/or bi-cistronic reporter plasmid containing Cap-Rluc-vIRES-Fluc. Plasmid construction, transcription in vitro and transfection using Lipofectamine 2000 reagent (Life Technologies) were done as previously described[19]. LPS (\#tlrl-peklps, InvivoGen) pre-incubation or post-treatment were specified in figure legends. Observation of cell morphology was performed with microscope. The detail information about cell culture, virus preparation and virus infection and luciferase assay referred to other reports [19]

\section{Western blot and antibodies}

The RD whole-cell lysates were prepared by lysing with RIPA buffer and western blot was performed as Wang et al described [20].Anti-EV-A71 VP1 (\#PAB7631-D01P) and anti- $\beta$-actin (\#BE0021-1000) were obtained from Abnova and EASYBIO, respectively. Anti-ERK1/2(\#9102), anti-phospho-ERK1/2 (\#9101) and anti- eIF4GI (\#2858) were purchased from Cell Signaling Technology. The target protein and $\beta$-actin were detected with anti-rabbit or mouse secondary antibody conjugated with horseradish peroxidase (\#BE0103-100 and \# BE0108-100, EASYBIO). Specific bands were visualized with enhanced chemiluminescent substrate (ECL).Each immunoblot assay was carried out at least three times and one of them was presented.

\section{Quantification of BactDNA fragments and EV-A71 RNA}


Quantification of BactDNA fragments was performed as previously described [21]. To avoid potentially bacterial contamination of molecular biology reagents, all specimens were processed in airflow chambers by the same investigator and all tubes were never exposed to free air. To remove potentially confounding $16 \mathrm{~S}$ rDNA contamination, 6 tubes of prepared DEPC water were set as negative controls and the processes of water from DNA extraction to quantitative PCR (qPCR) were completely synchronized with those of blood.

Genomic DNA was isolated from a total of $200 \mu \mathrm{L}$ of serum with QIAmp DNA Blood Minikit (Qiagen, Hilden, Germany) according to the manufacturer's instructions and DNA was eluted in a $100 \mu \mathrm{L}$ final volume. BactDNA levels were determined by $\mathrm{qPCR}$ in a $20 \mu \mathrm{L}$ amplification reaction with forward primer (5' -AGAGGGTGATCGGCCACA-3 ' ) and reverse primer (5 ' TGCTGCCTCCCGTAGGAGT-3 ' ), the universal eubacterial primers of a conserved region of $16 \mathrm{~S}$ rDNA gene. The amplification conditions for the 59 base pairs of DNA fragment were $95^{\circ} \mathrm{C}$ for $10 \mathrm{~min}$, followed by 45 cycles at $95^{\circ} \mathrm{C}$ for $15 \mathrm{~s}$ and $60^{\circ} \mathrm{C}$ for $60 \mathrm{~s}$. Each sample was amplified in triplicate and the BactDNA content was calculated according to a standard curve that generated from serial dilutions of plasmid DNA containing known copy numbers of the template. The final circulating BactDNA concentration was calculated by subtracting proportion of $16 \mathrm{~S}$ rDNA copies $/ \mu \mathrm{L}$ detected in water controls from those in blood. 
and expressed as the mean $(\mathrm{M}) \pm$ standard deviation $(\mathrm{SD})$ or number $(\%)$.Normal no outliers in continuous data by inspection of related boxplots. For comparison of demographic information and clinical characteristics at baseline among groups,

Fisher's exact Chi-square test or one-way analysis of variance (ANOVA) were conducted except specification. Analysis of covariance (ANCOVA) controlling for age and gender was used to analyze cytokines and bacterial measures among the three groups, and Bonferroni's multiple comparison test that can calculate the corrected statistical significance for multiple comparisons was performed for post-hoc analysis $P$-value $<0.05$ was accepted as the cutoff for statistical significance.

\section{Results} prone to have high body temperature, increased heart rate, elevated counts of white 
rashes in severe patients resembled those in mild patients, whereas erythematous and/or papulovesicular eruptions (atypical rashes) more frequently occurred in the mild $(10.77 \%$ vs. $1.54 \%, P<0.05)$ and herpangina was more common in the severe (64.62\% vs. $21.54 \%, P<0.01)$. Of the severe patients, the most common complication was neurological dysfunction (78.46\%), followed by pulmonary disorders $(29.23 \%)$ and cardiovascular disorders $(6.15 \%)$. Furthermore, there were no differences in aspects of heart rate, body temperature and laboratory results between healthy children and mild cases (all $P>0.05$ ).

Table 1 Clinic and laboratory characteristics of healthy subjects and HFMD patients

\begin{tabular}{|c|c|c|c|}
\hline Items & $\begin{array}{c}\text { Healthy } \\
\text { control }(n=65)\end{array}$ & $\begin{array}{c}\text { Mild } \\
\operatorname{HFMD}(\mathrm{n}=65)\end{array}$ & $\begin{array}{c}\text { Severe } \\
\operatorname{HFMD}(n=65)\end{array}$ \\
\hline Gender (Female/Male) & $28 / 37$ & $29 / 36$ & $28 / 37$ \\
\hline Age (months) & $41.42 \pm 15.44$ & $39.75 \pm 23.81$ & $27.57 \pm 15.53 * * *$ \\
\hline EV-A71 positive, $\mathrm{n}(\%)$ & - & $65(100)$ & $65(100)$ \\
\hline Typical rashes $\ddagger$, n (\%) & - & $55(84.62)$ & $56(86.15)$ \\
\hline Hands & - & $50(76.92)$ & $56(86.15)$ \\
\hline Feet & - & $49(75.38)$ & $53(81.54)$ \\
\hline Mouth & - & $42(64.62)$ & $50(76.92)$ \\
\hline Buttock & - & $19(29.23)$ & $26(40.00)$ \\
\hline Atypical rashes\#, n (\%) & - & $7(10.77)$ & $1(1.54)^{*}$ \\
\hline Herpangina, $\mathrm{n}(\%)$ & - & $14(21.54)$ & $42(64.62)^{* *}$ \\
\hline Cardiovascular disorders, $\mathrm{n}(\%)$ & - & - & $4(6.15)$ \\
\hline Neurological disorders, n (\%) & - & - & $51(78.46)$ \\
\hline Pulmonary disorders, $\mathrm{n}(\%)$ & - & - & $19(29.23)$ \\
\hline Heart Rate (/min) & $123.5 \pm 15.23$ & $127.12 \pm 18.37$ & $144.62 \pm 18.04 * * *$ \\
\hline Body Temperature $\left({ }^{\circ} \mathrm{C}\right)$ & $37.45 \pm 0.85$ & $37.45 \pm 0.37$ & $38.38 \pm 0.62 * * *$ \\
\hline WBC Count $\left(10^{9} / \mathrm{L}\right)$ & $7.43 \pm 1.66$ & $8.78 \pm 1.78$ & $12.01 \pm 3.42 * * *$ \\
\hline Lymphocyte Count $\left(10^{9} / \mathrm{L}\right)$ & $3.22 \pm 1.16$ & $3.65 \pm 1.40$ & $4.23 \pm 3.28$ \\
\hline Monocyte Count $\left(10^{9} / \mathrm{L}\right)$ & $0.54 \pm 0.25$ & $0.57 \pm 0.24$ & $0.81 \pm 0.62 * *$ \\
\hline Platelet Count $\left(10^{9} / \mathrm{L}\right)$ & $239.37 \pm 53.62$ & $254.92 \pm 49.27$ & $277.37 \pm 57.85 * *$ \\
\hline CK (U/L) & $102.37 \pm 47.83$ & $106.64 \pm 52.45$ & $112.23 \pm 104.35$ \\
\hline
\end{tabular}


ALT(IU/L)

AST (IU/L)

LDH (U/L)

Sampling time $(\mathrm{d}) \dagger$
$22.20 \pm 6.15$

$32.53 \pm 15.36$

$261.24 \pm 46.53$

$23.37 \pm 8.53$

$25.53 \pm 13.28$

$36.35 \pm 20.25$

$292.43 \pm 78.53$

$276.25 \pm 54.53$

$2.51 \pm 0.82$

$2.77 \pm 0.91$

\$Maculo-papular and/or vesicular rashes. \# Erythematous and/or papulovesicular eruptions. †Timing of serum sampling after onset of HFMD. Compared with mild HFMD group, $* P<0.05, * * P<0.01$, *** $P<0.001$. WBC, white blood cells; CK, creatine kinase; AST, aspartate transaminase; ALT, alanine aminotransferase; LDH, lactate dehydrogenase. The data were presented as number of patients (\%) or $\mathrm{M} \pm \mathrm{SD}$.

\section{Pro-inflammation phenotype dominates in HFMD cases}

As shown in Fig. 1, the results of ANCOVA analysis displayed that there were statistically significant differences between the healthy group, the mild and severe HFMD groups $\quad$ in $\quad$ terms of $\mathrm{CRP}(F=138.5, P<0.001)$, IL-1 $\beta(F=361.4, P<0.001)$, IL-6 $(F=276.1, P<0.001)$, IFN- $\gamma(F=730.9, P<0.001)$ and TNF- $\alpha(F=832.4, P<$ 0.001). Further, post-hoc analysis using Bonferroni's multiple comparison test found that serum levels of the inflammatory biomarkers dramatically increased approximately 3 - to 8 - times on average in the severe group in comparison with the mild group (all $P<0.001$ ), while the protein levels of sera CRP, IL-6, IFN- $\gamma$ and TNF- $\alpha$ in mild HFMD group were only 1- to 2-fold higher as compared to health control group (all $P<0.05$ ). These results demonstrate and verify the existence of systemic pro-inflammation in EV-A71-associated HFMD cases, especially in the severe patients. 

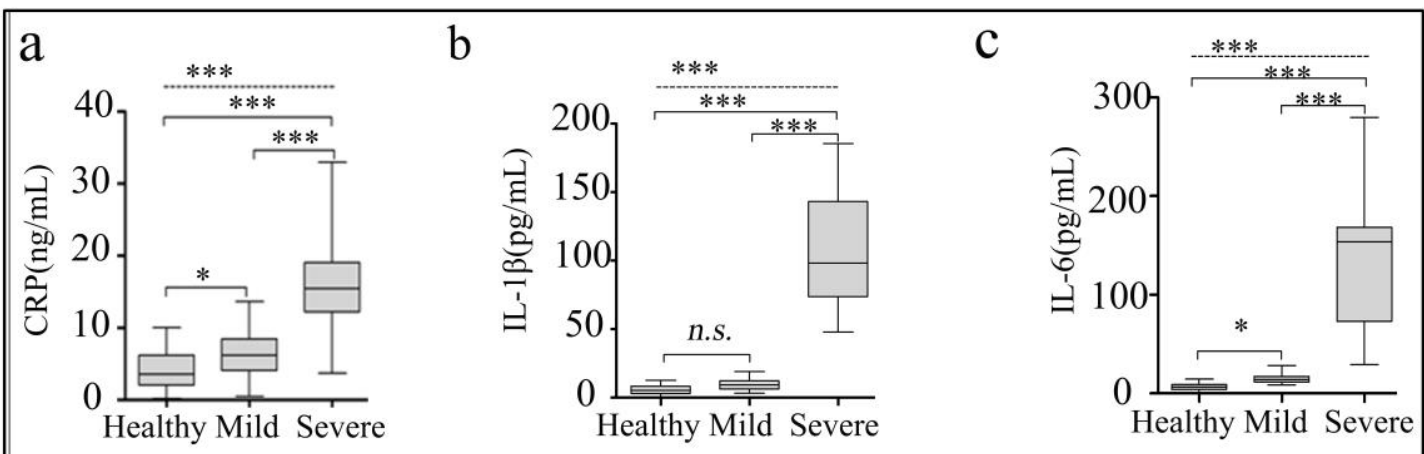

d

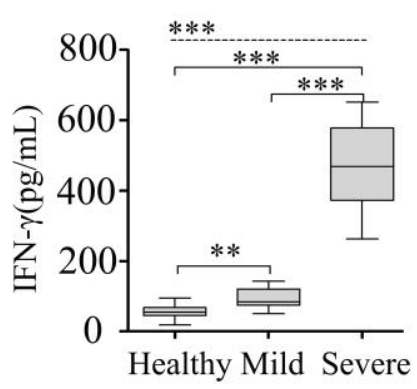

e

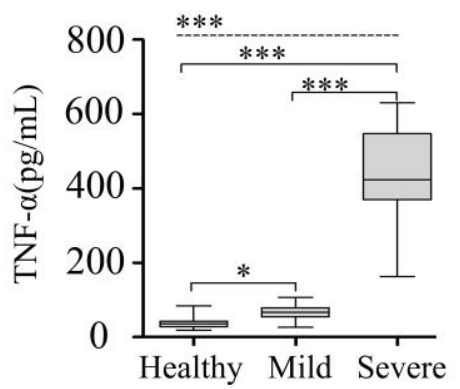

Fig.1 Differentially displayed cytokines in the three groups.

251 CRP, C-reactive protein; IL, interleukin; TNF, tumor necrosis factor; IFN, interferon.

Data were presented as boxplots. In post-hoc analysis using Bonferroni's multiple

253 comparison test, n.s. $>0.05, * P<0.05, * * P<0.01, * * * P<0.001, \cdots \cdots$ analysis using ANCOVA.

\section{BT occurs in severe HFMD cases}

Next, related serum markers of BT were measured in all the subjects (Fig.2). products (sCD14 and EndoCAb), and "leaky gut" (Claudin-3 and I-FABP), statistically significant differences between the three groups were all observed (all $P$

$261<0.01)$ from ANCOVA analysis results. Post-hoc analysis showed that only 

concentrations of LPS $(41.48 \pm 16.78$ vs. $19.44 \pm 8.81 \mathrm{pg} / \mathrm{mL}, \quad P<0.001), \quad$ sCD14 $\left(3.32 \pm 1.35\right.$ vs. $\left.1.47 \pm 1.12 \times 10^{6} \mathrm{pg} / \mathrm{mL}, \quad P<0.001\right)$, Claudin-3 $(42.53 \pm 20.48$ vs.
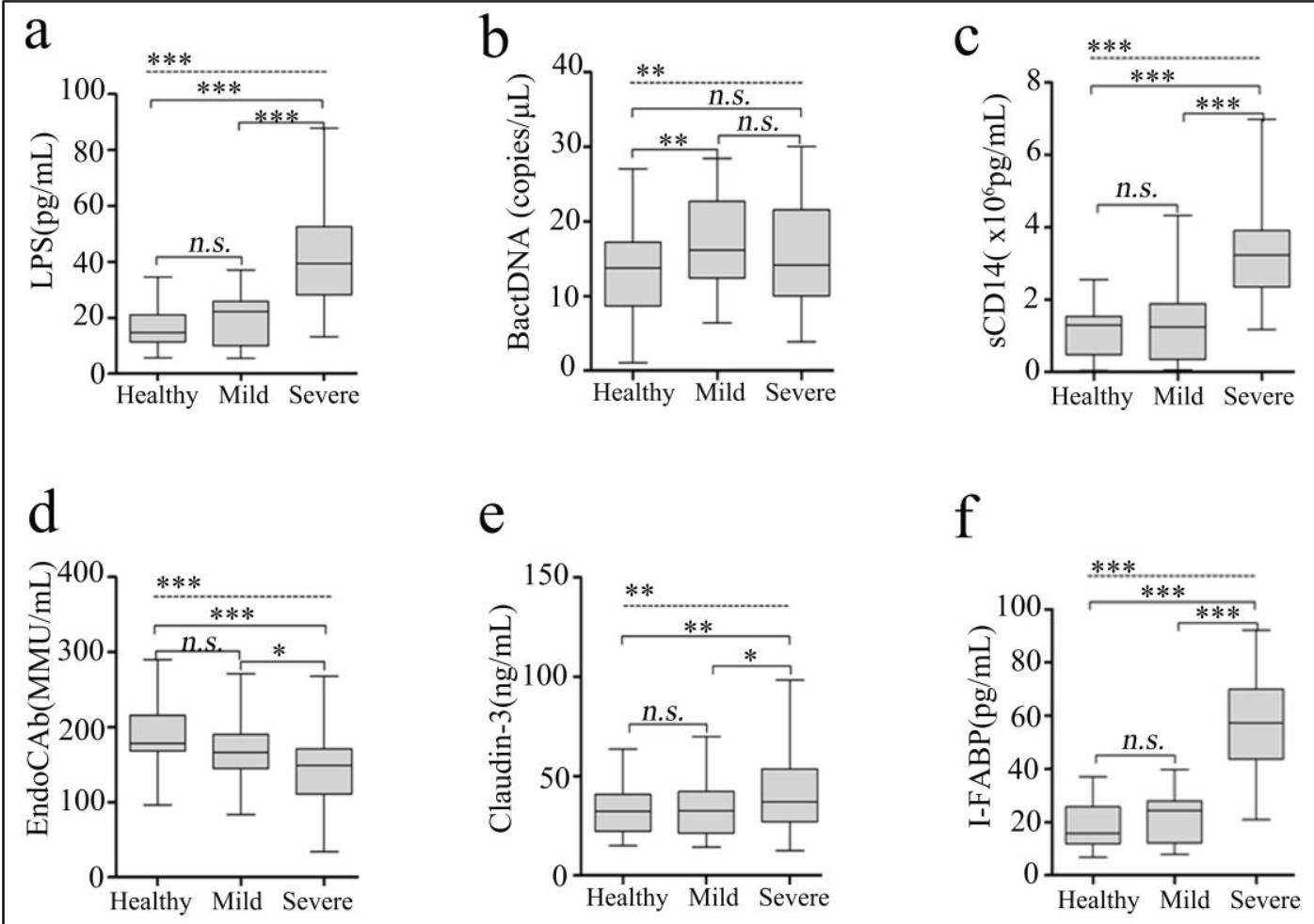

e

f
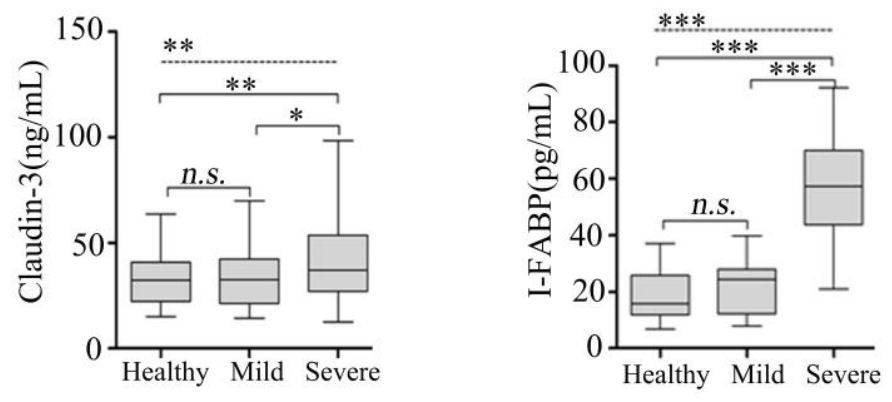

Fig. 2 BT-related biomarkers among groups of HFMD and healthy control.

274 LPS, lipopolysaccharide; BactDNA, bacterial DNA; sCD14, soluble CD14;

EndoCAb, endotoxin core antibody; I-FABP, intestinal fatty acid-binding protein. 
Data were presented as boxplots. In post-hoc analysis using Bonferroni's multiple comparison test, n.s. $>0.05, * P<0.05, * * * P<0.001$ analysis using ANCOVA

\section{LPS positively correlates with inflammation severity and serum viral protein}

In the severe group (Table 2), circulating concentration of LPS was further found to be positively correlated with all the quantified inflammatory mediators $(P<0.05$ for all variables); $\mathrm{sCD} 14$ was positively associated with $\mathrm{CRP}(P=0.041), \mathrm{IL}-1 \beta(P=0.001)$, IL-6 $(P=0.004)$ and IFN- $\gamma(P=0.019)$ after controlling potential confounders. Pro-inflammation was well-proved to facilitate viral replication in vivo and in vitro. In severe HFMD cases, serum protein levels of EV-A71 VP1 determined by ELISA were found to be positively correlated with serum LPS $[r=0.341, P=0.005$, Fig. 3a] and serum $\mathrm{sCD} 14[r=0.458, P<0.001$, Fig. $3 \mathrm{~b}]$, respectively. These data imply the link that circulating LPS from BT, as well as LPS responded SCD14, might be the important cause synergistically leading to the higher levels of pro-inflammation mediators and viral proteins observed in severe HFMD patients.

\begin{tabular}{lccccccccccc}
\hline & \multicolumn{2}{c}{ CRP } & \multicolumn{2}{c}{ IL-1 $\beta$} & \multicolumn{2}{c}{ IL-6 } & \multicolumn{2}{c}{ IFN- $\gamma$} & \multicolumn{2}{c}{ TNF- $\alpha$} \\
\cline { 2 - 10 } Cytokines & $r$ & $P$ & $r$ & $P$ & $r$ & $P$ & $r$ & $P$ & $r$ & $P$ \\
\hline LPS & 0.323 & $0.039^{*}$ & 0.470 & $0.002^{*}$ & 0.621 & $0.007^{*}$ & 0.319 & $0.041^{*}$ & 0.652 & $0.001^{*}$ \\
BactDNA & 0.122 & 0.341 & 0.237 & $0.037^{*}$ & 0.258 & 0.633 & 0.053 & 0.346 & 0.324 & 0.092 \\
I-FABP & 0.072 & 0.098 & 0.156 & 0.481 & 0.217 & 0.093 & 0.143 & 0.071 & 0.264 & 0.271 \\
\hline
\end{tabular}




\begin{tabular}{lccccccccccc}
\hline Claudin-3 & 0.051 & 0.365 & 0.224 & 0.472 & 0.232 & 0.053 & 0.204 & 0.094 & 0.044 & 0.431 \\
sCD14 & 0.672 & $0.041^{*}$ & 0.534 & $0.001^{*}$ & 0.648 & $0.004^{*}$ & 0.513 & $0.019 *$ & 0.837 & 0.052 \\
EndoCAb & 0.155 & 0.362 & 0.243 & 0.129 & 0.047 & 0.325 & 0.474 & 0.061 & 0.235 & 0.353 \\
\hline
\end{tabular}

$293 * P<0.05$. Analyses using partial correlation analysis.

294

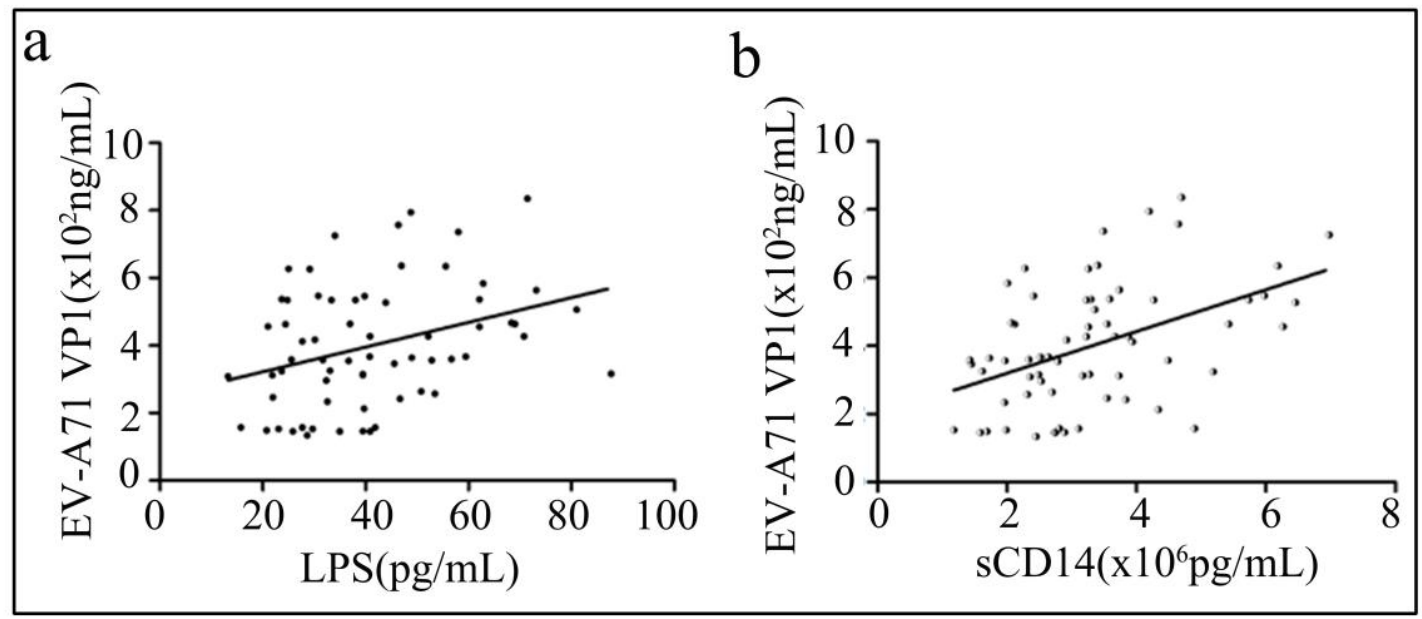

295

296

Fig.3 Partial correlation analysis of serum protein levels of EV-A71 VP1 and LPS

297 (a) or SCD14 (b) in severe HFMD cases.

298

2995 LPS pre-incubation slightly promote EV-A71 attachment and internalization

300

To explore the effect of circulating LPS on EV-A71 infection, EV-A71 Strain

$301 \mathrm{BrCr}$ was used to infect RD cells in vitro after pre-incubating the virus with $E$-coli

302 K12-derived LPS at $37^{\circ} \mathrm{C}$ for 2 hours. Firstly, cytotoxicity of LPS to RD cells was

303 determined and results found that LPS at less than $1 \mu \mathrm{g} / \mathrm{mL}$ was not toxic to the cells

304 (Fig. 4a). As Fig. 4 showed, only slightly increased levels of EV-A71 RNA on cell

305 surface (relative multiple:1.29 $\pm 0.13, P<0.05$ ) and that entering the cell (relative

306 multiple: $1.20 \pm 0.06, P<0.01$ ) were only observed in $500 \mathrm{ng} / \mathrm{mL}$ LPS treatment group 
in comparison with mock treatment group, which indicates that LPS pre-incubation only slightly facilitate EV-A71 infection at steps of viral attachment and internalization.

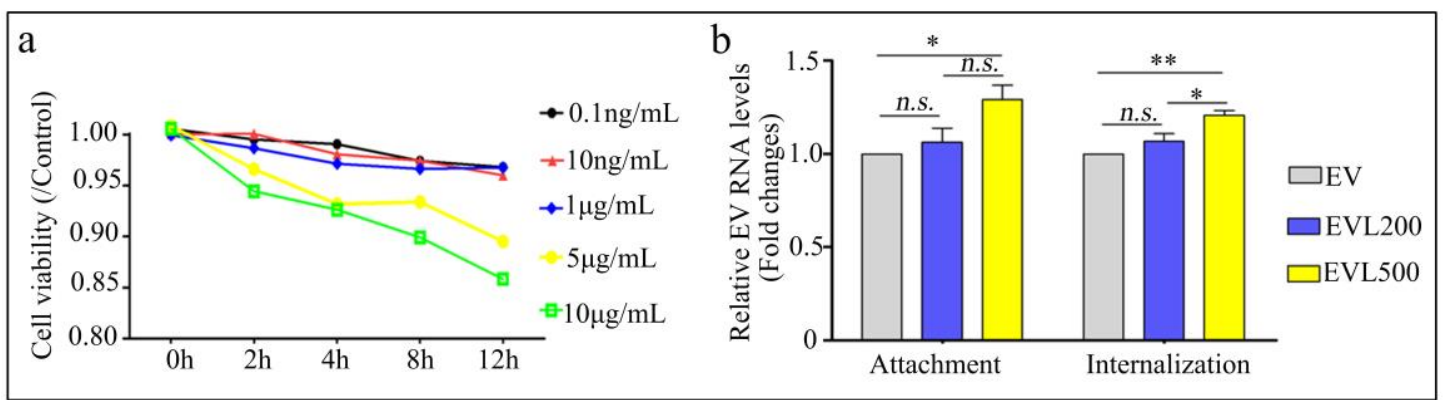

Fig. 4 LPS pre-incubation on EV-A71 attachment and internalization.

RD cells were treated with $0.1 \mathrm{ng} / \mathrm{mL}$ to $10 \mu \mathrm{g} / \mathrm{mL}$ LPS for 0 to 12 hours followed by cell viability assessment using CCK8 (a). 2 MOI of EV-A71 were pre-incubated with $200 \mathrm{ng} / \mathrm{mL}$ or $500 \mathrm{ng} / \mathrm{mL}$ LPS at $37^{\circ} \mathrm{C}$ for 2 hours, then the virus were used to infect RD cells with binding buffer on ice. 1 hour later, the cells were washed (attachment assessment) or/and cultured at $37^{\circ} \mathrm{C}$ for another 1 hour and then treated with trypsin (internalization assessment). Viral RNA was extracted by commercial kit and EV-A71 RNA was determined by qPCR (b). Data were showed as $\mathrm{M} \pm \mathrm{SD}$ and student's $t$ test was used for comparisons, n.s. $>0.05, * P<0.05, * * P<0.01$.

\section{LPS accelerate EV-A71 replication by promoting viral $2 \mathrm{~A}^{\text {pro }}$-mediated IRES activity}

The effect of LPS post-treatment on EV-A71 proliferation in vitro was further examined. Compared to virus-infected cells without LPS challenge, $200 \mathrm{ng} / \mathrm{mL}$ or 
500ng/mL LPS treatments dramatically promoted the occurrence of cytopathic effect (CPE) of RD cells induced by EV-A71 infection [Fig. 5a] and increased the expressions of VP1 [Fig. 5b]. In parallel, protein levels of LPS responded sCD14 in cellular supernatants were also increased with the treatments of LPS [Fig. 5c]. Because the synthesis of enterovirus protein is mediated by viral $2 \mathrm{~A}^{\text {pro }}$-driven viral IRES, effect of LPS post-treatment on viral IRES was assessed. As Fig. 5d presented, overexpression of viral $2 \mathrm{~A}^{\text {pro }}$ or treatment with $500 \mathrm{ng} / \mathrm{mL}$ LPS moderately promoted IRES activity $(P<0.05$ for all variables) compared with mock treatments, however, IRES activity was remarkably increased by $200 \mathrm{ng} / \mathrm{mL}$ or $500 \mathrm{ng} / \mathrm{mL}$ LPS in $2 \mathrm{~A}^{\text {pro }}$ overexpressed cells when compared with $2 \mathrm{~A}^{\text {pro }}$ or LPS treatment $(P<0.001$ for all variables). We previously proved that $2 \mathrm{~A}^{\text {pro }}$-driven viral IRES activity was regulated by cellular phosphorylated extracellular signal-regulated kinase (ERK)-mediated eIF4GI trans-cleavage [23]. Fig. 5e showed that $2 \mathrm{~A}^{\mathrm{pro}}$-mediated phosphorylation of ERK and cleavage of eIF4GI were significantly accelerated by $500 \mathrm{ng} / \mathrm{mL}$ LPS. These data collectively demonstrate that LPS can facilitate EV-A71 replication by promoting viral $2 \mathrm{~A}^{\mathrm{pro}}$-mediated IRES activity, which imply the contributory role of translocating LPS to the severity of EV-A71-induced HFMD. 


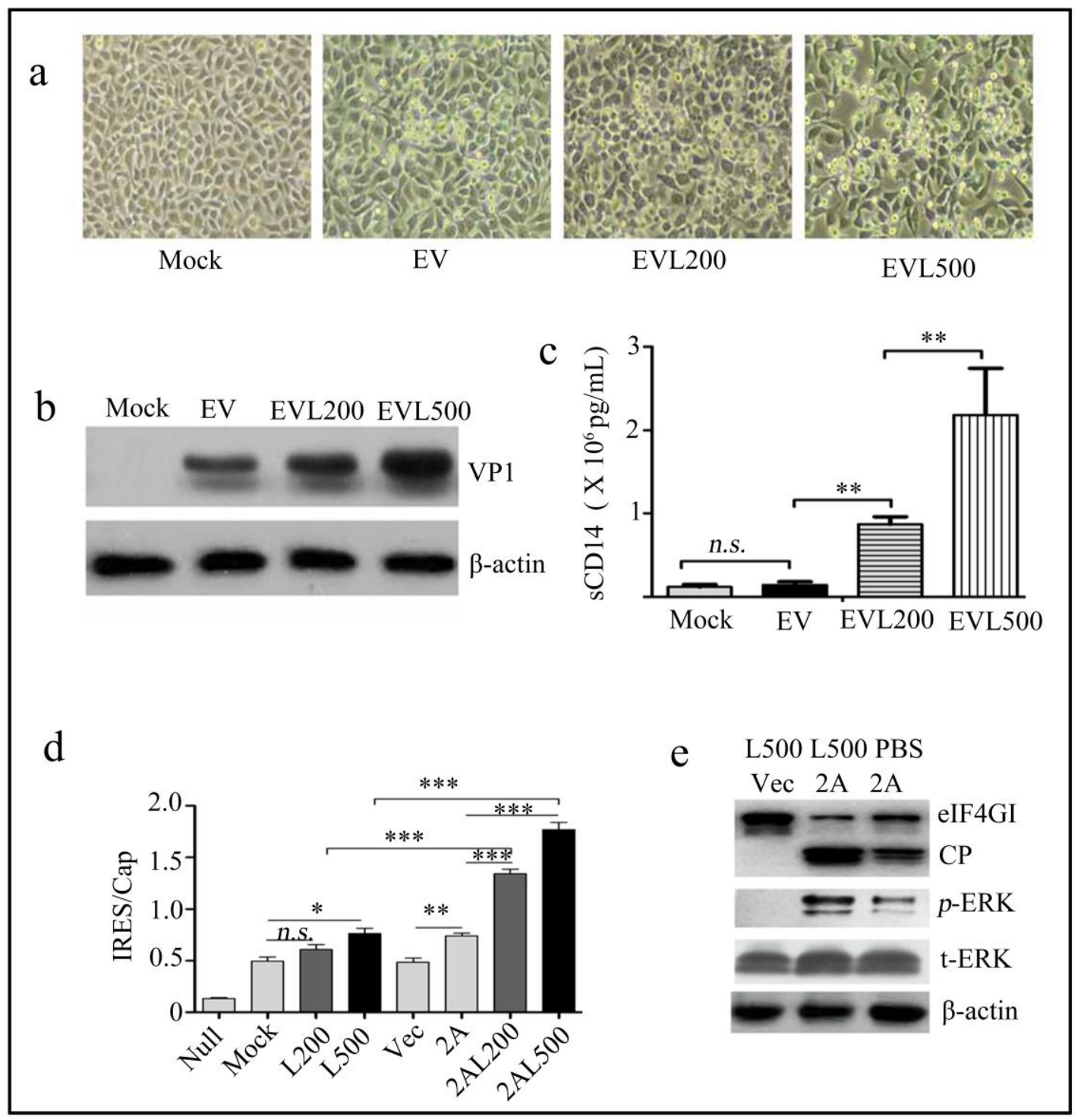

345 Fig.5 The effect of LPS post-treatment on proliferation of EV-A71 in vitro. RD cells

346 were infected with EV-A71 at an MOI of 2. 2 hours later, the cells were treated with

347 LPS at the concentration of $200 \mathrm{ng} / \mathrm{mL}$ or $500 \mathrm{ng} / \mathrm{mL}$, respectively. At 12 hours post

348 infection, photomicrographs were taken (original magnification, $100 \mathrm{X})(\mathrm{a})$, the protein

349 levels of viral VP1 in cell lysates were measured by western blot (b) and protein

350 levels of sCD14 in cellular supernatants were determined by ELISA(c). RD cells were

351 pre-transfected with p-EGFP-Vector (Vec, $2 \mu \mathrm{g} /$ well, 6-well plate) or p-EGFP-2A (2A,

$3522 \mu \mathrm{g} /$ well, 6-well plate), respectively. Subsequently, $12 \mathrm{~h}$ later, the cells were 
353

354

355

re-transfected with Cap-Rluc-vIRES-Fluc mRNA (100 ng/well, 96-well plate). 4 hours later, the cells were treated with $200 \mathrm{ng} / \mathrm{mL}$ or $500 \mathrm{ng} / \mathrm{mL}$ LPS for another 12 hours. The intensities of Fluc and Rluc were detected as described in MMs. The results (Rluc/Fluc) indicate the $\mathrm{M} \pm \mathrm{SD}$ of three independent experiments (d). $\mathrm{RD}$ cells were pre-transfected with p-EGFP-Vector (Vec) or p-EGFP-2A (2A), respectively. $12 \mathrm{~h}$ later, the cells were treated with $500 \mathrm{ng} / \mathrm{mL}$ LPS for another 12 hours. The cell lysates were used for the protein detection of eIF4GI, phosphorylated-ERK (p-ERK) and total ERK (t-ERK) by western blot (e). EV, EV-A71; EVL200, EV-A71+200ng/mL LPS; EVL500, EV-A71+500ng/mL LPS. 2AL200, 2A+200ng/mL LPS; 2AL500, 2A+500ng/mL LPS. Statistical difference was determined by student's $t$ test. n.s. $>0.05, * P<0.05,{ }^{*} * P<0.01, * * * P<0.001$.

\section{Discussion}

EV-A71 is generally regarded as the major causative agent for severe HFMD cases with neurological complications. Several independent studies have previously shown a close association between elevated inflammatory mediators and HFMD severity [8, 9, 24-26]. Consistent with that, levels of inflammatory mediators including CRP, IL-6, IFN- $\gamma$, IL-1 $\beta$ and TNF- $\alpha$ in mild and severe HFMD cases with EV-A71 infection in present study were moderately and dramatically increased, respectively. Serum IL-6 has been reported to be strongly associated with aseptic meningitis among children with EV-A71-induced HFMD [26].As is well known, to remove pathogenic microorganisms and protect the tissue from damage, CRP rises sharply in the plasma 
with IL-6 stimulation. IFN- $\gamma$, an imperative contributor of the generation of IFN- $\gamma$-inducible protein-10 (IP-10), is responsible for the recruitment of Th1 lymphocyte into the central nerve system during EV-A71 infection [8]. Although functional redundancies of IL-1 $\beta$ and TNF- $\alpha$ have been reported, the supposed pyrogenic role that contributes to the febrile response commonly observed in severe HFMD patients would be more crucial [9]. Actually, the increases in production of these inflammatory mediators were unlikely to be purely due to increased viral replication, they may interact and synergize to induce tissue damage in a sophisticated and coordinated network [8]. Previous profiling studies mainly explored the changes in inflammatory cytokines and potential cytokines for predicting the severity and criticality of HFMD. In present study, the involvement of inflammation response was further verified, more than that, we were particularly interested in the potential causes for systemic inflammation activation and we focused on the BT in EV-A71-associated HFMD individuals, which had never been investigated before.

BT is the passage of bacterium and/or bacterial products from the gut lumen into the organism in absence of bacteremia. In this process, increased intestinal mucosal permeability is indispensable. The tight junction is universally demonstrated to be the structural basis for maintaining normal intestinal permeability [27, 28]. Claudin-3 and I-FBPA, the two key components of tight junction, present at high levels in the blood can reliably reflect increased intestinal permeability as they are released into systemic circulation by enterocytes when intestinal epitheliums are compromised.

In present study, the evidence that remarkable increases in serum levels of 
I-FABP and Cludin-3 only in severe cases links the increased intestinal permeability with the severity of EV-A71-associated HFMD, which has not been reported previously. Correspondingly, the peripheral blood concentration of LPS, but not BactDNA, was significantly higher in severe other than mild cases with EV-A71 infection. Translocating LPS is in fact related to an exacerbation of the inflammatory response [29] and the following correlation analysis also showed that the circulating concentrations of inflammatory mediators had good correlativity with LPS, as well as sCD14. As LPS-specific host response, sCD14 circulates at high levels in the serum and interacts with translocating LPS to stimulate antigen-presenting cells via toll-like receptor 4 (TLR4) signaling [30].Under bacteria or LPS challenge, vascular endothelial cells and perivascular mast cells have been reported to express abundant TLR4, thus, the inflammatory cytokines are synthesized and secreted [31-33]. Furthermore, decreased host EndoCAb in peripheral blood failed to bind and clear LPS from circulation, which ensures high serum level of LPS for a long time and subsequently maintains systemic inflammation. It is also worth noting that serum BactDNA loads in mild cases with aggression may have little effect on inflammation state given the results from correlation analysis and differential expressions of BactDNA among mild or severe cases. We can only speculate that serum BactDNA loads quantified by qPCR likely underestimate the presence of BactDNA within whole blood and corresponding perturbation of inflammation markers may be transient. Collectively, these findings emphasize that translocating LPS is implicated in EV-A71-induced systemic inflammation responses and argue for a causative 
relationship between circulating LPS and disease exacerbations.

Most inflammatory cytokines are crucial immune modulators in host-virus interaction. Upon viral infection, the fine-tuning levels of myriad inflammatory mediators usually determine an anti-viral state advantageous to the hosts or a pro-viral state advantageous to the invading viruses. Translocating LPS will undoubtedly aggravate inflammatory response and may correspondingly promote viral propagation. Clinically, LPS, as well as sCD14, was demonstrated to be positively correlated with EV-A71 VP1 loads in serum in present study. Intestinal bacterial surface LPS was uncovered to bind poliovirus (a member of Enterovirus genus) and thus enhanced virion stability and cell attachment [34], which may be also exploited by EV-A71 for replication and transmission. In vitro, we further demonstrated that EV-A71 attachment and internalization were only slightly promoted by LPS pre-incubation; in contrast to that, EV-A71 proliferation was significantly facilitated by LPS post-incubation, which was further proved to be linked with viral $2 \mathrm{~A}^{\mathrm{pro}}$-mediated IRES activity. Apart from the mentioned perspectives, LPS was also proved to stimulate early growth response-1 (EGR1) translocation into the nucleus and the nuclear EGR1 facilitates EV-A71 replication by binding to EV-A71 5'UTR, a region that contains IRES structure [35].

Unfortunately, at least four limitations exist in our study. First, correlations between these inflammatory mediators and BactDNA in mild patients were not conducted as they were moderately elevated in comparison to the healthy children. Second, we didn't perform stratified analyses in subgroups of neurological 
441

442

dysfunction or cardio-respiratory disorders, which is partly to blame for the limited enrollment of severe cases. Furthermore, as with all case-controlled clinical studies, present study failed to adequately explain the causal relationship between BT and disease severity, related animal experiments are expected for ethical considerations. Last but not the least, the molecular mechanism by which translocating LPS promotes systemic inflammation and aids viral replication remains to be further investigated.

\section{Conclusion}

Current study mainly verifies the presence of leaky gut-caused bacterial translocation and further correlates translocating LPS to severity of EV-A71-induced HFMD possibly by driving pro-inflammation response and promoting viral $2 \mathrm{~A}^{\mathrm{pro}}$-mediated IRES activity. Collectively, these observations indicate that bacterial translocation may be a novel anti-inflammatory or antiviral therapeutic target for improving disease outcome in severe cases with EV-A71 infection.

\section{Acknowledgements: none}

\section{Abbreviations}

Lipopolysaccharide:LPS; EV-A71:Enterovirus A71; HFMD:hand, foot, and mouth disease; IRES:internal ribosomal entry site; IFN- $\gamma$ :interferon-gamma; CRP:C-reactive protein; TNF- $\alpha$ :tumor necrosis factor- $\alpha$; I-FABP:intestinal fatty acid binding protein; sCD14:soluble CD14; EndoCAb:endotoxin core antibody; eIF4GI:eukaryotic 
463 initiation factor 4GI; CPE:cytopathic effect; ERK:extracellular signal-regulated

464 kinase ;TLR4:toll-like receptor 4; EGR1:early growth response-1.

465 Funding

466 This work was funded by the grant from the National Natural Science Foundation of

467 China (82003337) and China Postdoctoral Science Foundation (2020M683268).

468

469

Declarations

470

Ethics approval and consent to participate

471 This study was approved by and carried out under the guidelines of the Ethics

Committee of Chongqing University Three Gorges Hospital.

473

Competing interests: None.

475

\section{Authors' contributions}

477

JY and BC designed the study; YW wrote the manuscript; QY and XF collected serum samples; XX and KD carried out the experiments; XY analyzed the data. All authors

479 read and approved the final manuscript.

480

Availability of data and materials

482 All data involved in this study is available upon reasonable request made to the corresponding author. 


\section{References}

1 Schmidt NJ, Lennette EH, Ho HH. An apparently new enterovirus isolated from patients with disease of the central nervous system. J Infect Dis,1974,129(3):304-309.

2 Li Y, Chang Z, Wu P, et al. Emerging Enteroviruses Causing Hand, Foot and Mouth Disease,

492

493

494

495

496

497

498

499

500

501

502

503

504

505 China, 2010-2016. Emerg Infect Dis,2018, 24(10):1902-1906.

$3 \mathrm{Yu} \mathrm{H}$, Cowling BJ.Remaining challenges for prevention and control of hand, foot, and mouth disease. Lancet Child Adolesc Health,2019, 3(6):373-374.

4 Head JR, Collender PA, Lewnard JA, et al. Early Evidence of Inactivated Enterovirus 71 Vaccine Impact Against Hand, Foot, and Mouth Disease in a Major Center of Ongoing Transmission in China, 2011-2018: A Longitudinal Surveillance Study. Clin Infect Dis, 2020,71(12):3088-3095.

5 Guo H, Li Y, Liu G, et al. A second open reading frame in human enterovirus determines viral replication in intestinal epithelial cells. Nat Commun, 2019,10(1):4066.

6 Yuan J, Shen L, Wu J, et al. Enterovirus A71 Proteins: Structure and Function. Front Microbiol,2018, 9:286.

7 Chen KR, Ling P. Interplays between Enterovirus A71 and the innate immune system. J Biomed Sci,2019, 26(1):95.

8 He Y, Feng Z, Wang W, et al. Global cytokine/chemokine profile identifies potential progression prediction indicators in hand-foot-and-mouth disease patients with Enterovirus A71 infections. Cytokine, 2019, 123:154765. 
9 Teo FMS, Nyo M, Wong AA, et al. Cytokine and Chemokine Profiling in Patients with Hand, Foot and Mouth Disease in Singapore and Malaysia. Sci Rep,2018, 8(1):4087.

$10 \mathrm{Li} \mathrm{W}$, Zhu Y, Li Y, et al. The gut microbiota of hand, foot and mouth disease patients demonstrates down-regulated butyrate-producing bacteria and up-regulated inflammation-inducing bacteria. Acta Paediatr, 2019, 108(6):1133-1139.

11 Stehle JR Jr, Leng X, Kitzman DW et al. Lipopolysaccharide-binding protein, a surrogate marker of microbial translocation, is associated with physical function in healthy older adults. J Gerontol A Biol Sci Med Sci, 2012, 67(11):1212-1218.

12 Hunt PW, Sinclair E, Rodriguez B, et al. Gut epithelial barrier dysfunction and innate immune activation predict mortality in treated HIV infection. J Infect Dis, 2014, 210(8):1228-1238.

13 Alvarez-Silva C, Schierwagen R, Pohlmann A, et al. Compartmentalization of Immune Response and Microbial Translocation in Decompensated Cirrhosis. Front Immunol,2019, 10:69.

14 Sikora M, Stec A, Chrabaszcz M, et al.Intestinal Fatty Acid Binding Protein, a Biomarker of Intestinal Barrier, is Associated with Severity of Psoriasis. J Clin Med,2019, 8(7):1021.

15 Luchetti MM, Ciccia F, Avellini C, et al.Gut epithelial impairment, microbial translocation and immune system activation in inflammatory bowel disease-associated spondyloarthritis.2020 [published online ahead of print]. Rheumatology (Oxford).

16 Marchetti G, Cozzi-Lepri A, Tincati C, et al.Immune activation and microbial translocation in liver disease progression in HIV/hepatitis co-infected patients: results from the Icona Foundation study. BMC Infect Dis,2014, 14:79.

17 Sandler NG, Koh C, Roque A, et al.Host response to translocated microbial products predicts 
outcomes of patients with HBV or HCV infection. Gastroenterology,2011, 141(4):1220-1230.

$18 \mathrm{Li}$ XW, Ni X, Qian SY, et al.Chinese guidelines for the diagnosis and treatment of hand, foot and mouth disease (2018 edition). World J Pediatr,2018, 14(5):437-447.

19 Chen B, Wang Y, Pei X, et al. Cellular Caspase-3 Contributes to EV-A71 2Apro-Mediated Down-Regulation of IFNAR1 at the Translation Level. Virol Sin, 2020,35(1):64-72.

20 Wang W, Xiao F, Wan P, et al.EV71 3D Protein Binds with NLRP3 and Enhances the Assembly of Inflammasome Complex. PLoS Pathog,2017, 13(1):e1006123.

21 Such J, Francés R, Muñoz C, et al. Detection and identification of bacterial DNA in patients with cirrhosis and culture-negative, nonneutrocytic ascites. Hepatology,2002, 36(1):135-141.

22 Gao M, Duan H, Liu J, et al. The multi-targeted kinase inhibitor sorafenib inhibits enterovirus 71 replication by regulating IRES-dependent translation of viral proteins. Antiviral Res, 2014,106:80-5.

23 Duan H, Zhu M, Xiong Q, et al. Regulation of enterovirus 2A protease-associated viral IRES activities by the cell's ERK signaling cascade: Implicating ERK as an efficiently antiviral target. Antiviral Res, 2017,143:13-21.

24 Zeng M, Zheng X, Wei R, et al.The cytokine and chemokine profiles in patients with hand, foot and mouth disease of different severities in Shanghai, China, 2010. PLoS Negl Trop Dis,2013, 7(12):e2599.

25 Sun JF, Li HL, Sun BX.Correlation analysis on serum inflammatory cytokine level and neurogenic pulmonary edema for children with severe hand-foot-mouth disease. Eur J Med Res,2018, 23(1):33.

26 Lee JY, Son M, Kang JH et al. Serum interleukin-6 levels as an indicator of aseptic meningitis 
551

552

553

554

555

556

557

558

559

560

561

562

563

564

565

566

567

568

569

570

571

572

among children with enterovirus 71-induced hand, foot and mouth disease. Postgrad Med,2018, 130(2):258-263.

27 Nagpal R, Yadav H. Bacterial Translocation from the Gut to the Distant Organs: An Overview. Ann Nutr Metab,2017, 71(Suppl 1):11-16.

28 Rooks MG, Garrett WS.Gut microbiota, metabolites and host immunity. Nat Rev Immunol,2016, 16(6):341-352.

29 Murtaj V, Belloli S, Di Grigoli G, et al. Age and Sex Influence the Neuro-inflammatory Response to a Peripheral Acute LPS Challenge. Front Aging Neurosci,2019, 11:299.

30 Tsukamoto H, Takeuchi S, Kubota K, et al.Lipopolysaccharide (LPS)-binding protein stimulates CD14-dependent Toll-like receptor 4 internalization and LPS-induced TBK1-IKK€-IRF3 axis activation. J Biol Chem,2018, 293(26):10186-10201.

31 Page AV, Liles WC.Biomarkers of endothelial activation/dysfunction in infectious diseases. Virulence,2013, 4(6):507-516.

32 Zeuke S, Ulmer AJ, Kusumoto S et al.TLR4-mediated inflammatory activation of human coronary artery endothelial cells by LPS. Cardiovasc Res,2002, 56(1):126-134.

33 Jin $\mathrm{Y}$, Zhang C, Wang H, et al.Mast cells contribute to Enterovirus 71 infection-induced pulmonary edema in neonatal mice. Lab Invest,2018, 98(8):1039-1051.

34 Robinson CM, Jesudhasan PR, Pfeiffer JK.Bacterial lipopolysaccharide binding enhances virion stability and promotes environmental fitness of an enteric virus. Cell Host Microbe,2014, 15(1):36-46.

35 Song Y, Cheng X, Yang X, et al. Early growth response-1 facilitates enterovirus 71 replication by direct binding to the viral genome RNA. Int J Biochem Cell Biol,2015, 62:36-46. 